\title{
A 3D Optical Metamaterial Made by Self-Assembly
}

\author{
Silvia Vignolini ${ }^{1 *}$, Nataliya A.Yufa ${ }^{1 *}$, Pedro S. Cunha ${ }^{1}$, Stefan Guldin ${ }^{1}$, Ilia Rushkin ${ }^{2}$, Morgan \\ Stefik $^{3}$, Kahyun Hur ${ }^{3}$, Ulrich Wiesner ${ }^{3}$, Jeremy J. Baumberg ${ }^{1}$ \& Ullrich Steiner ${ }^{1}$
}

\begin{abstract}
Optical metamaterials are materials which, due to their mesostructure, have optical characteristics not found in nature. While demonstrated at microwave and yellow frequencies, optical metamaterials in higher frequencies require the assembly of three-dimensional architectures with structure control on the $\mathbf{1 0}-\mathbf{n m}$ length scale. Here we demonstrate such a truly three-dimensional optical metamaterial, generated by filling with metal a scaffold made by self-assembly of a block copolymer. The final gold structure has features which are two orders of magnitude smaller than the wavelength of visible light. As a result, it behaves as the archetypal Pendry wire metamaterial with linear and circular dichroism.
\end{abstract}

The prediction and subsequent creation of artificially engineered metamaterials has opened the pathway to revolutionary effects in light-matter interactions ${ }^{1-6}$. In such materials the properties of the dielectric and magnetic permittivities $\epsilon$ and $\mu$ are not governed by the response of the individual atoms in the presence of an electromagnetic field, but are determined by the sub-wavelength structure of the material. In 1996 Pendry et al. predicted that a sparse cubic metal wire array with micron-wide wires would have a significantly reduced plasma frequency due to the reduced average electron density and the large selfinductance of the structure ${ }^{2}$.

A plethora of experimental work in this field has demonstrated metamaterials from $\mathrm{GHz}^{7}$ up to yellow optical frequencies $^{8}$. Reaching higher optical frequencies has proven problematic as it requires the manipulation of materials on the scale of just a few tens of nanometres over macroscopic areas. While fabrication techniques such as focused ion beam lithography, direct laser writing and atomic layer deposition $^{9-13}$ provide design flexibility, they are limited with regard to accessible feature sizes as well as the scalability of samples.

Until now, colloidal self-organisation was the only type of self-assembly used to create metamaterials ${ }^{14}$. These materials have feature sizes limited to above several hundreds of nanometres and to sphere-packing geometries. Block copolymers (BCPs) are unique in offering a route to creating macroscopically large samples with a variety of complex 3D architectures with features on the nanometre length scale. Unlike earlier work based on BCPs, ${ }^{15}$ we incorporate a continuous metal phase into a $\mathrm{BCP}$ scaffold ${ }^{16}$, thereby producing the first truly optical, threedimensional metamaterial. As predicted by Pendry et $a l^{2}$, this material has a reduced plasmon frequency. In addition, it exhibits strongly anisotropic plasmon modes with
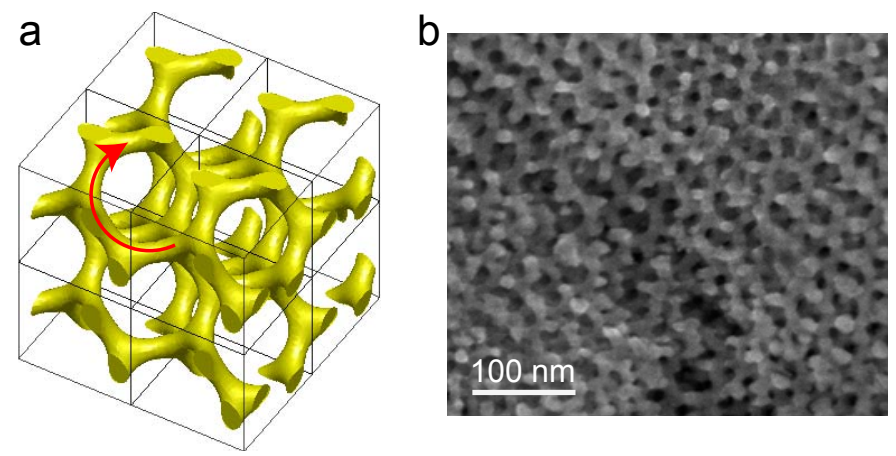

Figure $1 \mid$ Gyroid morphology. a, A computer-simulated view of the single gyroid. The arrow indicates the handedness along the [111] axis. b, SEM image of a metal gyroid by filling a porous polymer network.

linear dichroism and optical chirality across the visible region.

BCPs are polymers which consist of two or more distinct polymer chains (blocks) joined by a covalent bond. The interplay between the energetic penalty for stretching the blocks is balanced by the chemical repulsion between the blocks, creating a range of morphologies such as spheres, cylinders and gyroids ${ }^{17}$. Increasing the number of blocks leads to an increase in the number of accessible morphologies $^{18}$.

There has been previous work using BCP scaffolds with dielectrics for optical applications ${ }^{19}$. In this work, however, we opt to replace one of the continuos nanoscale polymer networks with a metal in order to create a novel material with interesting optical properties very different from those of a structured dielectric. Such replication is possible because the blocks in BCPs are chemically distinct, hence we can selectively etch them and then backfill with inorganic materials to replicate self-assembled structures into materials that are not amenable to direct selfassembly in such complex morphologies.

Here we demonstrate the creation of a three-dimensional gold metamaterial based on BCP self-assembly. We start with an isoprene-block-styrene-block-ethylene oxide (ISO) $\mathrm{BCP}$ which forms two chemically distinct, interpenetrating gyroid networks $(\mathrm{I}, \mathrm{O})$ of opposite chirality in a matrix of the third block ${ }^{20}$. The I gyroid network is then removed by selective UV/chemical etching and backfilled with gold by electrodeposition. The final device consists of a continuous, triply periodic network of gold (Fig. 1). The dimension of the full unit cell is $\sim 50 \mathrm{~nm}$, far below

\footnotetext{
${ }^{1}$ Cavendish Laboratory, Department of Physics, University of Cambridge, J. J. Thomson Avenue, Cambridge CB3 OHE, UK.

${ }^{2}$ Department of Mathematics, University of Nottingham, University Park, Nottingham NG7 2RD, UK. ${ }^{3}$ Department of Materials Science \& Engineering, Cornell University, Ithaca, New York 14853.

*These authors contributed equally to this work.
} 

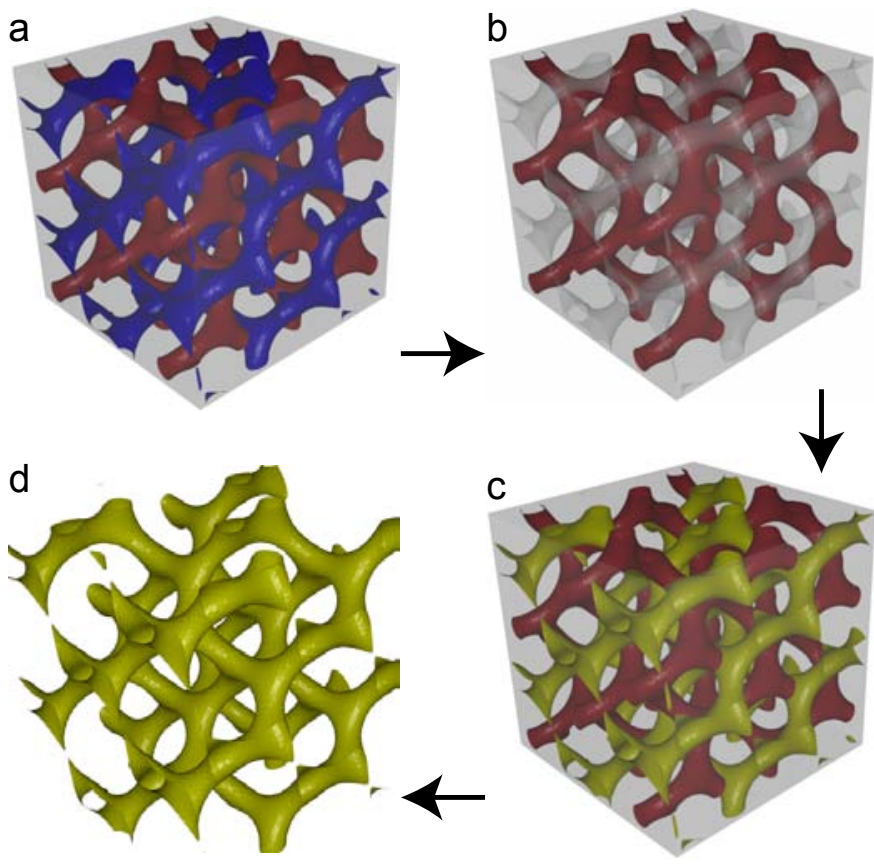

Figure $2 \mid$ Schematic representation of sample fabrication. a, The three different colours (red, blue and grey) correspond to the different blocks of the copolymer. The isoprene block (blue) is removed in $\mathbf{b}$ and is then back-filled with gold (yellow) in c. The final structure is obtained by plasma etching the two remaining polymer blocks, revealing a $3 \mathrm{D}$ continuous gold network in air in $\mathbf{d}$.

optical wavelengths. This particular morphology has been chosen since it is predicted to offer a strong resonant response which depends on the relative orientation between the structure and the polarisation of the incident light. Figure 1a shows the [110] projection of the gyroid.

Samples were prepared using isoprene-block-styreneblock-ethylene oxide (ISO) BCP films which are known to self-assemble into the gyroid morphology with a chiral isoprene phase, schematically shown in Fig. 2. We utilised a poly(isoprene- $b$-styrene- $b$-ethylene oxide) (ISO) prepared by sequential anionic polymerization using standard methods ${ }^{18,21}$. The molecular weight of the polyisoprene block was determined by GPC; the molecular weights of the polystyrene and poly(ethylene oxide) blocks by NMR, and the final polydispersity was determined by GPC. ISO used in this study was composed of 14.63, 29.04, and $9.77 \mathrm{~kg} / \mathrm{mol}$ of I, S, and O, respectively, with a molecular weight of $53.44 \mathrm{~kg} / \mathrm{mol}$ and a polydispersity of 1.05 . A detailed characterisation of this polymer shows selfassembly into the alternating gyroid phase ${ }^{20}$. Polymer film scaffolds were prepared by blade-coating a $5 \% \mathrm{w} / \mathrm{v}$ ISO anisole solution onto glass substrates with a conducting coating for electrodeposition. The resulting films were exposed to UV light $(254 \mathrm{~nm})$ for 3 hours and rinsed with ethanol to remove the isoprene. The resulting pore net-
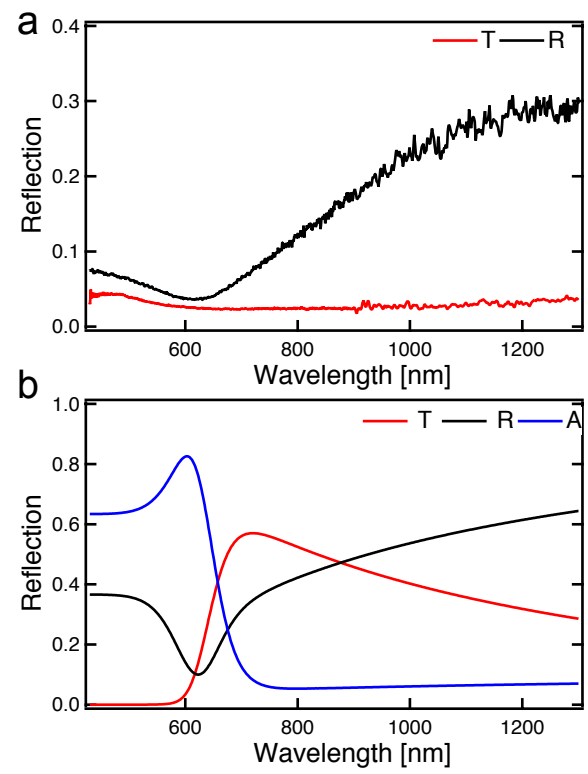

Figure 3 | Transmission and reflection spectra for unpolarised incident light. a, Exprimental data compared with b, the finite difference time domain calculations of a full 3D representation of the gyroid.

work was backfilled with gold by electrodeposition, to a thickness of $200 \mathrm{~nm}$. Thicker samples have also been prepared and will be the subject of a future study. In the electrochemical setup, a saturated calomel electrode was used as the reference. The deposition consisted of a nucleation and a deposition step. The nucleation step consisted of 1-3 cyclic voltammetry scans between $0 \mathrm{~V}$ and $-1.2 \mathrm{~V}$ at a scan rate of $50 \mathrm{mV} / \mathrm{s}$. The deposition step at a fixed potential of $-0.8 \mathrm{~V}$ for $100 \mathrm{~s}$ resulted in a final thickness of about $200 \mathrm{~nm}$. The remaining polymer was removed by plasma etching.

The samples were spectroscopically characterised using a white-light-illuminated optical microscope with fibre detection in a confocal configuration. (see Methods). In Fig. 3 the experimental results for both reflection and transmission configurations with unpolarised incident light are compared with the results of calculation of the ideal structures using a finite difference time domain code ${ }^{22}$ employing the material parameters for gold ${ }^{23}$. In the comparison, we have to take into account that an $\approx 5 \mathrm{~nm}$ thick solid $\mathrm{Au}$ layer at the bottom of the gyroid layer that is not included in the calculation. The presence of this layer partially reduces the transmission of the fabricated $\sim 200 \mathrm{~nm}$ thick sample. A simple Maxwell-Garnett averaging of the refractive index of the $\mathrm{Au} /$ air composite with the same $\mathrm{Au}$ fill fraction of $f=21 \%$ predicts near-perfect transmission in the red and infrared, completely opposite to the observation of Fig. 3. In fact, the presence of an ordered and continuos nm-scale gyroid network results in a reduction of the plasma frequency of gold. This is also confirmed by 

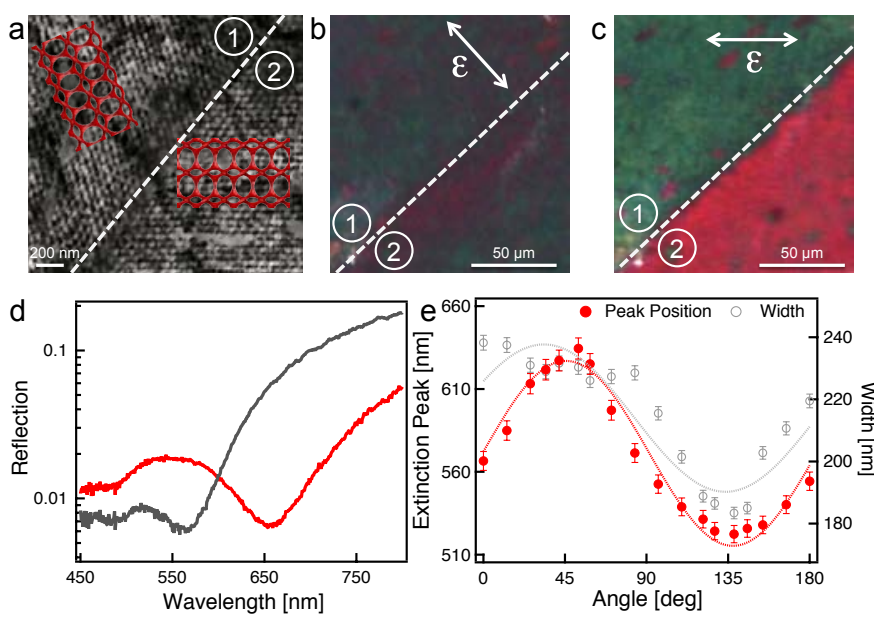

Figure $4 \mid$ Linear dichroism of the Au gyroid. a, SEM image of the area where the optical measurements were performed. b,c, Optical reflection images obtained with linearly polarised illumination, indicated by white arrows. No optical contrast is observed for a polarisation perpendicular to the grain boundary (b), while a polarisation parallel to the gyroid [100] direction of one of the domains produces the largest optical contrast (c). d, Reflection spectra for Area 1 with incident polarisation directions perpendicular (red) and parallel (grey) to the [100] gyroid direction. e Variation in extinction (1-reflectivity) peak position and width as a function of the angle between [100] gyroid direction and incident light polarisation.

finite difference time domain calculations. The qualitative agreement between the spectra in Fig. 3 show a decrease in reflection at about $600 \mathrm{~nm}$ that can be explained in terms of the Drude model in a reduction of plasma frequency from the bulk value of $7.5 \mathrm{eV}$ to $1.55 \mathrm{eV}$. Thus, the composite gyroid material behaves like a new metal with its own distinct optical characteristics: a plasma edge at $750 \mathrm{~nm}$ and a transparency which is greatly increased compared to any naturally-occurring bulk metal.

Two contributions are responsible for the modified plasma wavelength, $\lambda_{\mathrm{p}}=\lambda_{\mathrm{p}}^{\mathrm{Au}} \sqrt{m_{\mathrm{eff}} / f}$ (where $m_{\mathrm{eff}}$ is the effective mass of the electrons in the actual material), from that of $\mathrm{Au}\left(\lambda_{\mathrm{p}}^{\mathrm{Au}}=165 \mathrm{~nm}\right)$. The network of Au struts surrounded by air reduces the net electron density and accounts for about half of the reduction in the plasma frequency. The other contribution comes from the increased self-inductance of the structure produced when multiplyconnected wires are formed, which span all three spatial axes.

The SEM image in Fig. 4a reveals the morphology of the sample on the length scale of tens of microns. While the single gyroid has the same orientation perpendicular to the substrate surface across the entire sample, twinning in lateral directions is found, with domain sizes of 10$100 \mathrm{\mu m}$. The angle between the [100] direction at the twin- ning boundary is approximately $105^{\circ}$. Since the structure is anisotropic, its optical response strongly depends on the gyroid orientation. The investigated samples were therefore marked using a micromanipulator, in order to precisely relate the spectroscopic measurements to the twinning morphology obtained by SEM.

Imaging and spectroscopy in reflection under linearly polarised illumination show a clear birefringence across each twinned gyroid domain, observed as strong colour changes in Fig. 4b,c and supplementary movie S1. When the grain boundary is perpendicular to the polarization direction, light is coupled equally into the two twinned domains, which results in identical reflected colour (Fig. $4 \mathrm{~b})$. Since the twinning angle between the two regions is $105^{\circ}$, when the polarisation of the incident light is parallel to the [100] direction of one of the areas it is nearly perpendicular to the [100] direction of its twin, resulting in different colours in reflection (Fig. 4c, movie S1). Two different reflection spectra are observed in the orthogonal directions (Fig. 4d), with an admixture between them as the polarisation direction is varied. These reflection spectra show increasing absorption at shorter wavelengths with a peak which tunes sinusoidally with the polarisation direction between 525 and $630 \mathrm{~nm}$, shown in Fig. 4e for one of the domains. As well as this shift in the absorption peak position, the spectral linewidth of the absorption band is a function of the polarisation direction, tracking the observed spectral shifts. Performing the same analysis for the twin domain reveals a spectral modulation with the same period and amplitude but with a phase shift that is in perfect agreement with the twinning angle between the domains.

Light polarised in different directions couples differently to localised plasmon resonances of the interconnected gold network. A simple way to understand this behaviour is to approximate the gyroid as a network of disconnected $\mathrm{Au}$ rods. In the gyroid sample, a short-wavelength resonance is seen for a polarisation along the [100] direction of the domain, which is red-shifted by $20 \%$ in the perpendicular direction. These observations are intuitively in agreement with the rod-like morphology of the structure at normal incidence, with the short-wavelength transverse mode of the rods matching that of $10 \mathrm{~nm} \mathrm{Au}$ nanoparticles $(520 \mathrm{~nm}$ ), and the long-wavelength longitudinal mode produced by the optical field along the rod-like structure.

To probe the gyrotropic behaviour of this metamaterial, transmission measurements on the same twinned domains as above were taken with left- and right-hand circularly polarised light. The sample was tilted by $35^{\circ}$ and rotated around the [110] surface normal. For each full turn there are two rotation angles for which the optical axis coincides with the chiral [111] direction of the gyroid, that is, the microscope "looks down" the unobstructed gyroid channels (inset in Fig. 5, supplementary movie S2). Figure 5 plots the normalised difference in gyrotropic transmission $\Delta T$ 


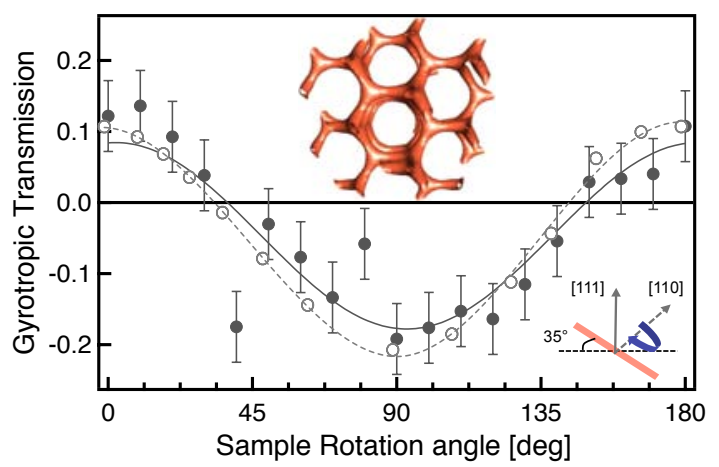

Figure 5|Gyrotropic transmission through a sample a inclined at $35^{\circ}$ The gyrotropic transmission is the difference between the transmission spectra in left and right channels as the sample was rotated around the [110] axis. The open symbols are the results of a finite difference time domain calculation. Lines are guides to the eye.

between right- and left-hand polarised light as a function of sample rotation angle, averaged over frequency region between 600 and $750 \mathrm{~nm}$ (where there is little spectral variation). The transmission of circularly polarised light is maximal for the two rotation angles $\left(0^{\circ}\right.$ and $\left.180^{\circ}\right)$ where the optical axis is co-linear with the [111] and [1 $1-1]$ directions. By varying the rotation angle, the optical path is increasingly obstructed by Au struts, reducing $|\Delta T|$. The rotation of the sample therefore leads to the clear observation of chirality in this gyroid metamaterial. The gyrotropic transmission is again very well reproduced by a finite difference time domain calculation.

We have thus successfully created the first selfassembled chiral optical metamaterial with 3D structure. This material is continuous, has features on the 10-nm length scale and exhibits orientation-dependent colour under linearly polarised incident light, as well as optical chirality. These findings pave the way for the potential mass production of optical metamaterials, which can lead to a variety of large-scale applications.

\section{METHODS SUMMARY}

The samples were prepared by using a triblock poly(isopreneblock-styrene-block-ethylene oxide) (ISO) copolymer to create several-micron-thick ordered films, which were slowly dried at room temperature. A detailed characterisation of the ISO block-copolymer used in this study confirms the formation of the chiral alternating gyroid morphology in the isoprene phase $^{20}$. The isoprene block was then removed by UV etching, and the resulting network of pores was back-filled with gold by electrodeposition from the conducting substrate, up to a thickness that could be varied between 200 and $1000 \mathrm{~nm}$. The remaining polymer was subsequently removed by plasma etching.

For optical characterisation, a 50- $\mu \mathrm{m}$ core optical fibre served as a pinhole in the conjugate to the focal plane of a $\times 10$ long working distance microscope objective, allowing collection of a signal with a spatial resolution below the sample domain dimensions. A broadband halogen lamp acted as the illumination source. Linear polarisation measurements were obtained using achromatic polarisers (Thorlabs, Inc.), and for circular polarisation measurements the polarisers were combined with super-achromatic quarter wave quartz plates (B.Halle Nachfl. $\mathrm{GmbH})$. The sample was mounted on a multi-rotational stage that allowed rotation around the focal axis of the objective and tilting away from normal incidence. This configuration enabled transmission and reflection measurements to be taken in different directions of the sample, including chiral gyroid directions. Self-consistent field theory was utilised to simulate a realistic triblock copolymer alternating gyroid morphology. The morphology of ISO was calculated, with relative block volume fractions of $0.28,0.57$, and 0.15 of I, S and O, respectively, using the simulation parameters of Tyler et al. ${ }^{24}$ and the I domain was extracted for the finite-difference time-domain simulations. Transmittance, reflectance and absorption of a $495 \mathrm{~nm}$ thick alternating gyroid film was simulated using a finite-difference time-domain simulator ${ }^{22}$. The material parameters for gold from Vial et al. were used $^{23}$.

1. Veselago, V. G. The electrodynamics of substances with simultaneously negative values of $\epsilon$ and $\mu$. Soviet Physics Uspekhi 10, 509 (1968).

2. Pendry, J. B., Holden, A. J., Stewart, W. J. \& Youngs, I. Extremely low frequency plasmons in metallic mesostructures. Phys. Rev. Lett. 76, 4773-4776 (1996).

3. Pendry, J., Holden, A., Robbins, D. \& Stewart, W. Magnetism from conductors and enhanced nonlinear phenomena. IEEE Transactions on Microwave Theory and Techniques 47, 2075 -2084 (1999).

4. Pendry, J. B. Negative refraction makes a perfect lens. Phys. Rev. Lett. 85, 3966-3969 (2000).

5. Pendry, J. B., Schurig, D. \& Smith, D. R. Controlling electromagnetic fields. Science 312, 1780-1782 (2006).

6. Leonhardt, U. Optical conformal mapping. Science $\mathbf{3 1 2}$, 1777-1780 (2006).

7. Smith, D. R., Padilla, W. J., Vier, D. C., Nemat-Nasser, S. C. \& Schultz, S. Composite medium with simultaneously negative permeability and permittivity. Phys. Rev. Lett. 84, 4184-4187 (2000).

8. Xiao, S. et al. Loss-free and active optical negative-index metamaterials. Nature 466, 735-8 (2010).

9. Plum, E., Fedotov, V. a., Schwanecke, a. S., Zheludev, N. I. \& Chen, Y. Giant optical gyrotropy due to electromagnetic coupling. Applied Physics Letters 90, 223113 (2007).

10. Clark, A. W., Glidle, A., Cumming, D. R. S. \& Cooper, J. M. Plasmonic split-ring resonators as dichroic nanophotonic DNA biosensors. Journal of the American Chemical Society 131, 17615-9 (2009).

11. Decker, M. et al. Strong optical activity from twisted-cross photonic metamaterials. Optics Letters 34, 2501-3 (2009). 
12. Güney, D. O., Koschny, T. \& Soukoulis, C. M. Intraconnected three-dimensionally isotropic bulk negative index photonic metamaterial. Optics Express 18, 12348-53 (2010).

13. Decker, M., Klein, M. W., Wegener, M. \& Linden, S. Circular dichroism of planar chiral magnetic metamaterials. Optics Letters 32, 856-8 (2007).

14. Lodewijks, K., Verelle, N., Van Roy, W., Borghs, G. \& Van Dorpe, P. Self-assembled hexagonal double fishnets as negative index materials. arXiv:1010.5138v1 [physics.optics] (2010).

15. Edrington, a. C. et al. Polymer-Based Photonic Crystals. Advanced Materials 13, 421-425 (2001).

16. Vukovic, I. et al. Supramolecular Route to Well-Ordered Metal Nanofoams. Acs Nano 5, 6339-6348 (2011).

17. Bates, F. S. \& Fredrickson, G. H. Block copolymers-designer soft materials. Physics Today 52, 32-38 (1999).

18. Epps, T. H. et al. Ordered network phases in linear poly(isoprene-b-styrene-b-ethylene oxide) triblock copolymers. Macromolecules 37, 8325-8341 (2004).

19. Hsueh, H.-Y. et al. Inorganic Gyroid with Exceptionally Low Refractive Index from Block Copolymer Templating. Nano letters 4994-5000 (2010).

20. Stefik, M. et al. Networked and chiral nanocomposites from abc triblock terpolymer coassembly with transition metal oxide nanoparticles (2011). In preparation.
21. Bailey, T., Pham, H. \& Bates, F. Morphological behavior bridging the symmetric $a b$ and $a b c$ states in the poly(styrene-b-isoprene-b-ethylene oxide) triblock copolymer system. Macromolecules 34, 6994-7008 (2001).

22. Oskooi, A. F. et al. A flexible free-software package for electromagnetic simulations by the fdtd method. Computer Physics Communications 181, 687-702 (2010).

23. Vial, A., Grimault, A.-S., Macías, D., Barchiesi, D. \& de la Chapelle, M. L. Improved analytical fit of gold dispersion: Application to the modeling of extinction spectra with a finitedifference time-domain method. Phys. Rev. B 71, 085416 (2005).

24. Tyler, C. A., Qin, J., Bates, F. S. \& Morse, D. C. Scft study of nonfrustrated abc triblock copolymer melts. Macromolecules 40, 4654-4668 (2007).

25. Wohlgemuth, M., Yufa, N., Hoffman, J. \& Thomas, E. L. Triply periodic bicontinuous cubic microdomain morphologies by symmetries. Macromolecules 34, 6083-6089 (2001).

Acknowledgements The authors thank J.J. Rickard, M. Scherer and A. Finnemore for their invaluable help. We acknowledge the EPSRC grant EP/G060649/1 for funding.

Author Information Correspondence and requests for materials should be addressed to J.B. (jjb12@cam.ac.uk) or U.S. (u.steiner@phy.cam.ac.uk). 


\section{Supplementary Information}

\section{Methods}

\section{Optical Characterisation}

A 50- $\mu \mathrm{m}$ core optical fibre served as a pinhole in the conjugate to the focal plane of a $\times 10$ long working distance microscope objective, giving a signal with a spatial resolution below the sample domain dimensions. A broadband halogen lamp acted as the illumination source. Linear polarisation measurements were obtained using achromatic polarisers (Thorlabs, Inc.). For circular polarisation measurements the polarisers were combined with super-achromatic quarter-wave plates (B. Halle Nachfl. GmbH). The sample was mounted on a stage that allowed rotation around the focal axis of the objective and tilting away from normal incidence. This enabled transmission and reflection measurements to be taken in different directions of the sample.

\section{Gyroid Visualization}

The level-set approximation to the gyroid surface is given by ${ }^{25}$

$$
\sin 2 \pi y \cos 2 \pi z+\sin 2 \pi z \cos 2 \pi x+\sin 2 \pi x \cos 2 \pi y=t,
$$

where the parameter $t$ depends on the volume fractions of the blocks.

\section{Finite Difference Time Domain Calculation}

Self-consistent field theory was utilised to simulate a realistic triblock copolymer alternating gyroid morphology. The morphology of ISO was calculated, with relative block volume fractions of $0.28,0.57$, and 0.15 of I, S and O, respectively, using the simulation parameters of Tyler et al. ${ }^{24}$ and the I domain was extracted for the simulations.

Transmittance, reflectance and absorption of a $495 \mathrm{~nm}$ thick alternating gyroid film was simulated using a finite-difference time-domain simulator ${ }^{22}$. The material parameters for gold from Vial et al. were used ${ }^{23}$. Bloch boundary conditions were applied along all three axes and perfectly matched layers were introduced along the film normal (see supplementary figure). The alternating gyroid metamaterial was excited by a continuous plane wave with linear or circular polarisation. Reflectance and transmittance were obtained by averaging the transmitted power, i.e. the Poynting vector, through the receiver planes. Systems without the metamaterial were also simulated for normalisation. The gyrotropic transmission was obtained by averaging the 640 to $710 \mathrm{~nm}$ wavelength range at an oblique angle incidence of $35^{\circ}$. 


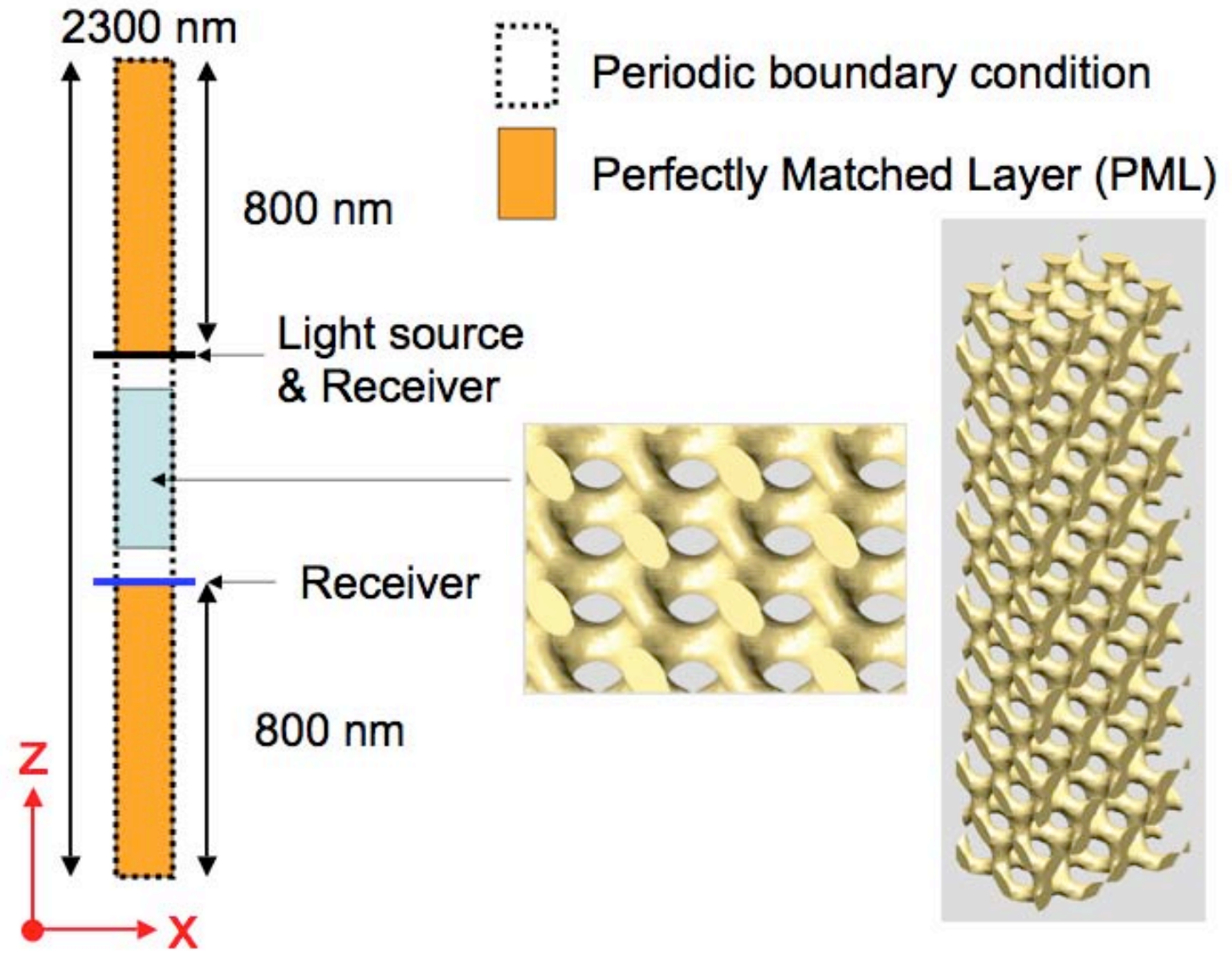

Supplementary Figure 1 | Schematic representation of the morphology used in the finite-difference time-domain simulation. Periodic boundary conditions were used and 800-nm-thick perfectly matched layers that absorb light were introduced in the top and bottom parts of the simulation system. Seven [110]-oriented unit cells of the alternating gyroid were stacked along $z$ direction to form a 495-nm-thick film. 